Research Paper

\title{
A comparative study of efficacy and safety of transarterial chemoembolization with CalliSpheres and conventional transarterial chemoembolization in treating unresectable intrahepatic cholangiocarcinoma patients
}

\author{
Tao Sun ${ }^{1,2,3 \#, ~ W e i h u a ~ Z h a n g ~}{ }^{1,2,3 \#, ~ L e i ~ C h e n ~}{ }^{1,2,3 \#, ~ Y a n q i a o ~ R e n ~}{ }^{1,2,3}$, Yiming Liu ${ }^{1,2,3}$, Chuansheng Zheng ${ }^{1,2,3 凶}$ \\ 1. Department of Radiology, Union Hospital, Tongji Medical College, Huazhong University of Science and Technology, Wuhan 430022, China. \\ 2. Hubei Province Key Laboratory of Molecular Imaging, Wuhan 430022, China. \\ 3. Department of Radiology, Union Hospital, Tongji Medical College and Wuhan National Laboratory for Optoelectronics, Huazhong University of Science and \\ Technology. \\ \#These authors contributed equally to this work. \\ $\triangle$ Corresponding author: Chuansheng Zheng and/or Yiming Liu; Department of Radiology, Union Hospital, Tongji Medical College, Huazhong University of Science and \\ Technology, Wuhan 430022, China. Email: hqzcsxh@sina.com. \\ (c) The author(s). This is an open access article distributed under the terms of the Creative Commons Attribution License (https://creativecommons.org/licenses/by/4.0/). \\ See http://ivyspring.com/terms for full terms and conditions.
}

Received: 2021.09.27; Accepted: 2022.01.01; Published: 2022.01.24

\begin{abstract}
Objective: Previous studies reported that drug-eluting beads transarterial chemoembolization (DEB-TACE) with CalliSpheres is effective and safe to treat hepatocellular carcinoma patients and metastatic liver cancer patients, however few studies reported its clinical application in intrahepatic cholangiocarcinoma (ICC) patients. Therefore, this study aimed to compare the efficacy between DEB-TACE versus conventional transarterial chemoembolization (cTACE) in unresectable ICC patients.

Methods: Between January 2016 and June 2020, 89 patients with unresectable ICC were retrospectively analyzed, and enrolled into DEB-TACE group $(\mathrm{N}=40)$ and cTACE group $(\mathrm{N}=49)$ based on the transarterial treatment. Treatment response was assessed according to modified Response Evaluation Criteria in Solid Tumors (mRECIST). Time to progression (TTP) and overall survival (OS) were analyzed by using the Kaplan-Meier curve. Factors affecting OS and TTP were determined by Cox's proportional hazards regression model.

Results: DEB-TACE group showed higher DCR (87.5\% vs. $65.3 \%, P=0.011)$, while similar ORR $(67.5 \%$ vs. $57.1 \%, P=0.317$ ) compared to CTACE group. Furthermore, DEB-TACE group had longer OS (median 10 months vs 6 months, $P=0.006$ ), while similar TTP compared to cTACE group (median 4 months vs 2 months, $P=0.098$ ). After adjustment by multivariant Cox's regression, DEB-TACE (versus CTACE) independently correlated with longer $O S(P=0.031)$. Further subgroup analyses displayed that $O S$ was prolonged in DEB-TACE group compared to CTACE group in patients with multiple tumors $(P=0.032)$ and patients with no lymph node metastasis $(P=0.023)$. Apart from abdominal pain, no difference of adverse events between the two groups was observed. There was no difference in liver function (Bilirubin, Albumin, Prothrombin time) before and after treatment (4 weeks) in both groups.
\end{abstract}

Conclusion: In patients with unresectable ICC, DEB-TACE significantly improved the OS when compared with cTACE and was well tolerated.

Key words: Intrahepatic cholangiocarcinoma, drug-eluting beads transarterial chemoembolization, overall survival, time to progression; treatment response

\section{Introduction}

Intrahepatic cholangiocarcinoma (ICC) is a biliary epithelium malignancy with a rapid increase of global incidence rate and mortality rate over last several decades $[1,16]$. However, Due to profound vascular invasiveness and distal metastasis, most ICC patients are in advanced stage when diagnosed and are not suitable for the curative options like surgical resection and transplant $[1,3]$. Even after the curative 
options, early recurrence and metastasis were common. Therefore, it is a need to develop novel therapies to improve survival in unresectable ICC patients.

Conventional transarterial chemoembolization (cTACE) is the most common intra-arterial modality in treating unresectable ICC patients [5]. However, minimal vascularity of the ICC and lower drug penetration due to loaded chemotherapeutic agent leakage remain huge concerns [6,7]. To overcome these drawbacks, drug-eluting beads transarterial chemoembolization (DEB-TACE) is an alternative technique which uses a non-absorbable microsphere to embolize in the arteries of target tumor and to simultaneously release the loaded therapeutic agent in a controlled manner $[6,7,8]$. In the past of 15 years, many drug-eluting beads (DEBs) have been produced, among which CalliSpheres microsphere is the first manufactured DEB in China with good loading and release profile [17]. In terms of its clinical application, DEB-TACE with CalliSpheres microsphere has formerly been applied in treating hepatocellular carcinoma (HCC) patients and gained promising efficacy with less safety issues compared to cTACE [9,10,11]. Furthermore, DEB-TACE with CalliSpheres microsphere also displays efficacy and safety in treating ICC patients $[12,13]$. However, to our best knowledge, no comparison between DEB-TACE using CalliSpheres microsphere and CTACE has been conducted in patients with unresectable ICC yet. Therefore, we conducted this study and aimed to compare the efficacy and safety between DEB-TACE with CalliSpheres microsphere and cTACE in unresectable ICC patients.

Patients with unresectable ICC received DEB-TACE or cTACE $(n=183)$

Excluded ( $\mathrm{n}=94)$

-Missing data $(\mathrm{n}=10)$

-EOGO $>1(n=4)$

-Mixed feature ICC-HCC (n=9)

-absence of liver biopsy or postoperative

pathological examinations $(\mathrm{n}=71)$

Patients with unresectable ICC were enrolled in the study $(n=89)$

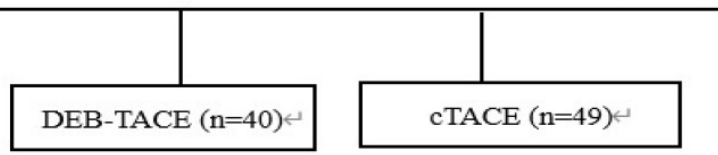

Figure 1. Flow chart. DEB-TACE: Drug-eluting beads transarterial chemoembolization, cTACE: conventional transarterial chemoembolization, ICC: intrahepatic cholangiocarcinoma.

\section{Methods}

\section{Study design and population}

This retrospective study was approved by Union Hospital, Tongii Medical College, Huazhong University of Science and Technology Review Board of our hospital. The informed consent from the patients were waive by the board because the study was a retrospective study. This study was conducted in accordance with the Declaration of Helsinki. A total of 89 patients with unresectable ICC treated by DEB-TACE or cTACE in our hospital between January 2016 and January 2019 were retrospectively analyzed. Clinical data of patients were reviewed, and the patients were considered eligible for analysis only if they met the following criteria: (i) diagnosed as unresectable ICC confirmed by liver biopsy (63 cases) or postoperative pathological examinations (26 cases); (ii) Child-Pugh stage A or B; (iii) Eastern Cooperative Oncology Group Performance Status (ECOG PS) score $\leq 1$ point; (iv) treated by CTACE or DEB-TACE with CalliSpheres microsphere; (v) complete clinical data and survival data; (vi) without other malignancies. Among 89 ICC patients, 40 patients received DEB-TACE with CalliSpheres microsphere and were divided into DEB-TACE group; 49 patients underwent cTACE and were divided into cTACE group for the analysis. Written informed consents were collected from all the patients.

\section{Clinical data collection}

For study analysis, the following preoperative clinical data were collected from the medical records systems: age, gender, hepatitis B virus (HBV) infection, ECOG PS score, Child-Pugh stage, tumor number, tumor size, lymph node metastasis, ascites, and biochemical indexes. In addition, TACE treatment information and previous treatments (such as surgery, and percutaneous transhepatic cholangial drainage/magnetic resonance cholangiopancreatography (PTCD/ MRCP)) were also collected.

\section{TACE procedure}

In brief, femoral artery puncture was performed by a micro-puncture system with a 5F vascular introducer (Cook, Bloomington, Indiana, USA). Celiac arteriography with or without superior mesenteric arteriography was implemented for evaluation of the feeding arteries of tumor, the arterial anatomy, as well as the patency status of the portal vein. Subsequently, superselective catheterization with a microcatheter was performed for the feeding arteries of tumor. 
The mixture of CalliSpheres microsphere (Jiangsu Hengrui Medicine Co. Ltd, Lianyungang, Jiangsu, China) and nonionic contrast medium (DEB-TACE group) or lipiodol (Lipiodol Ultrfluido, Guerbet, Paris, France) was injected into the tumor feeders through the microcatheter at a speed of $1 \mathrm{~mL} / \mathrm{min}$. In the DEB-TACE group, the CalliSpheres microsphere with diameter of $100-300 \mu \mathrm{m}$ was used to load $80 \mathrm{mg}$ epirubicin as chemoembolization agents. Drug-loading procedures of CalliSpheres microsphere were performed as the technological process described in previous study. In the cTACE group, 10-20 mL lipiodol (Lipiodol Ultrfluido, Guerbet, Paris, France) mixed with 20-40 mg epirubicin was used as chemoembolization agents. The injecting amount of mixture (CalliSpheres microsphere or lipiodol) was determined to the size of tumor. When the blood flow slowed or the small branch of portal vein appeared viewed by angiography, the TACE procedure stopped. The Polyvinyl Alcohol (PVA) could be added when it was necessary.

\section{Follow-up and assessments}

The first follow-up was administered at fourth week following the first TACE procedure, during which, the abdominal contrast-enhanced computed tomography (CT), or magnetic resonance (MRI) was carried out for assessment of tumor response. Patients were subjected to another TACE if there were residual lesions or progression dieses (PD) revealed in the examination of CT or MRI. After the first follow-up, subsequent visit was conducted every 2 months, which was performed until January 2020. The documents of response evaluation and follow-up were collected, analyzed and compared. Evaluation of tumor response was in accordance with the modified RECIST criteria on the basis of the medical imaging of the abdominal enhanced CT or MRI. Objective response rates (ORR) and disease control rate (DCR) were calculated as reported in the previous study. Time to progression (TTP) was defined as the time interval from the first TACE treatment to the first disease progression. Overall survival (OS) was defined as the time interval from the first TACE treatment to patient's death regardless of the causes, or the last visit. In addition, adverse events were collected and reported according to the National Cancer Institute Common Terminology Criteria for Adverse Events (version 4.0).

\section{Statistical analysis}

Data processing and analyzing were completed with the use of SPSS 24.0 (IBM, Armonk, New York, USA). For descriptive analysis, continuous variables and categorical variables were described as mean \pm standard deviation (SD) and frequency (percentage), respectively. The Pearson $\chi^{2}$ test, correction $\chi^{2}$ test, Fisher's exact test, and independent-sample $t$ test were applied to analyze the difference between two groups. Kaplan-Meier curve was plotted for displaying the profiles of accumulating TTP and OS. Estimation of difference in survival data between two groups was performed based on log-rank test. Variables with $P$ value $\leq 0.2$ in the univariate analysis were further included in the multivariate Cox's proportional hazards regression model analysis to assess the factors that affected TTP or OS. A $P$ value $<0.05$ (two-tailed) was considered as there was statistical significance in corresponding analysis.

\section{Results}

\section{Patients' clinical features}

From January 2016 to June 2020, 89 unresectable ICC patients received DEB-TACE (40 patients) or CTACE (49 patients) were enrolled in this study. Baseline characteristics were well balanced between two groups and were listed in Table 1 . The median follow-up period was 7 months (range, 2-19 months).

\section{Efficacy of DEB-TACE and CTACE for unresectable ICC}

In DEB-TACE group, there were no case with CR, 27 (67.5\%) cases with PR, 8 (20.0\%) cases with SD, and $5(12.5 \%)$ cases with PD (Table 2). In the cTACE group, there were no cases with CR, $28(57.1 \%)$ cases with PR, 4 (8.2\%) cases with SD, 18 (36.7\%) cases with PD. The ORR of the DEB-TACE group was $67.5 \%$, which was similar with the CTACE group $(57.1 \%$, $P=0.317)$. However, the DCR of DEB-TACE was significantly higher than CTACE group $(87.5 \%$ vs. $65.3 \%, P=0.011$ ). During the follow-up, all patients died in the both groups. Although there was no difference of accumulating TTP between DEB-TACE group (median 4 months, 95\% CI: $(2.6,5.3)$ months) and CTACE group (median 2 months, $95 \%$ CI: $(1.2,2.9)$ months) $(P=0.098)$ (Figure 2A), DEB-TACE group (median 10 months, 95\% CI: $(6.1,13.9)$ months) exhibited a longer accumulating OS compared to cTACE group (median 6 months, 95\% CI: $(4.5,7.5)$ months) $(P=0.006)$ (Figure 2B).

\section{Factors related to survival profile}

Univariant Cox's regression model analysis displayed that treatment (DEB-TACE vs. CTACE) was not correlated with TTP in ICC patients $(P=0.158)$ (Table 3). Furthermore, after adjustment by multivariant Cox's regression, treatment option (DEBTACE vs. cTACE) failed to independently predict TTP in ICC patients $(P=0.132)$. Univariant Cox's regression model analysis exhibited that treatment option 
(DEB-TACE vs. cTACE) correlated with longer OS in ICC patients $(\mathrm{P}=0.012)$ (Table 4). After adjustment by multivariant Cox's regression, treatment option (DEB-TACE vs. cTACE) could be an independent factor to predict longer OS in ICC patients $(P=0.031)$.

Table 1. Characteristics of patients

\begin{tabular}{|c|c|c|c|}
\hline Characteristics & $\begin{array}{l}\text { DEB-TACE } \\
(\mathrm{N}=40)\end{array}$ & $\begin{array}{l}\text { cTACE } \\
(\mathrm{N}=49)\end{array}$ & $P$ value \\
\hline Age (years), mean $\pm S D$ & $61.8 \pm 10.7$ & $57.4 \pm 10.4$ & 0.053 \\
\hline Gender, No. $(\%)$ & & & 0.608 \\
\hline Male & $25(62.5)$ & $28(57.1)$ & \\
\hline Female & $15(37.5)$ & $21(42.9)$ & \\
\hline HBV, No. (\%) & $3(7.5)$ & $5(10.2)$ & 0.943 \\
\hline ECOG PS score, No. $(\%)$ & & & 0.722 \\
\hline 0 & $17(42.5)$ & $19(38.8)$ & \\
\hline 1 & $23(57.5)$ & $30(61.2)$ & \\
\hline Child-Pugh stage, No. (\%) & & & 0.322 \\
\hline A & $35(87.5)$ & $39(79.6)$ & \\
\hline B & $5(12.5)$ & $10(20.4)$ & \\
\hline Tumor number, No. $(\%)$ & & & 0.512 \\
\hline Single & $16(40.0)$ & $23(46.9)$ & \\
\hline Multiple & $24(60.0)$ & $26(53.1)$ & \\
\hline Tumor size $(\mathrm{cm})$, mean $\pm \mathrm{SD}$ & $7.9 \pm 6.6$ & $7.0 \pm 4.1$ & 0.410 \\
\hline Lymph node metastasis, No. (\%) & $31(77.5)$ & $29(59.2)$ & 0.744 \\
\hline Ascites, No. $(\%)$ & $3(7.5)$ & $4(8.2)$ & 0.999 \\
\hline \multicolumn{4}{|l|}{ Biochemical indexes } \\
\hline $\operatorname{ALT}(\mathrm{U} / \mathrm{L})$, mean $\pm \mathrm{SD}$ & $41.8 \pm 53.2$ & $49.8 \pm 45.7$ & 0.454 \\
\hline $\operatorname{Albumin}(\mathrm{g} / \mathrm{L})$, mean $\pm S D$ & $35.5 \pm 5.1$ & $35.8 \pm 10.5$ & 0.375 \\
\hline Bilirubin (umol/L), mean \pm SD & $19.8 \pm 6.3$ & $19.3 \pm 4.7$ & 0.452 \\
\hline $\mathrm{PT}(\mathrm{s})$, mean $\pm \mathrm{SD}$ & $13.9 \pm 2.1$ & $13.7 \pm 1.9$ & 0.534 \\
\hline Neutrophile $\left(x 10^{9} / \mathrm{L}\right)$, mean \pm SD & $5.0 \pm 2.1$ & $4.3 \pm 2.1$ & 0.143 \\
\hline Lymphocyte $\left(x 10^{9} / \mathrm{L}\right)$, mean \pm SD & $1.1 \pm 0.5$ & $1.5 \pm 0.6$ & 0.002 \\
\hline $\operatorname{PLT}\left(\times 10^{9} / \mathrm{L}\right)$, mean \pm SD & $188.8 \pm 86.0$ & $190.4 \pm 84.7$ & 0.931 \\
\hline CA125 $(\mathrm{U} / \mathrm{mL})$, mean $\pm \mathrm{SD}$ & $131.5 \pm 204.8$ & $121.8 \pm 273.3$ & 0.853 \\
\hline CA199 $(\mathrm{U} / \mathrm{mL})$, mean \pm SD & $518.4 \pm 525.7$ & $528.5 \pm 1722.0$ & 0.972 \\
\hline TACE session, No. (\%) & & & 0.741 \\
\hline 1 & $19(47.5)$ & $25(51.0)$ & \\
\hline$\geq 2$ & $21(52.5)$ & $24(49.0)$ & \\
\hline \multicolumn{4}{|l|}{ Previous treatments } \\
\hline Surgery, No. (\%) & $8(20.0)$ & $18(36.7)$ & 0.084 \\
\hline Radiofrequency ablation, No. (\%) & $0(0)$ & $0(0)$ & - \\
\hline Systematic chemotherapy, No. (\%) & $4(10.0)$ & $6(12.2)$ & 0.739 \\
\hline Targetded therapy, No. (\%) & $0(0)$ & $0(0)$ & - \\
\hline PTCD/MRCP, No. (\%) & $9(22.5)$ & $15(30.6)$ & 0.391 \\
\hline \multicolumn{4}{|c|}{$\begin{array}{l}\text { DEB-TACE, drug-eluting beads transarterial chemoembolization; cTACE, } \\
\text { conventional transarterial chemoembolization; SD, standard deviation; HBV, } \\
\text { hepatitis B virus; ECOG PS, Eastern Cooperative Oncology Group Performance } \\
\text { Status; ALT, alanine aminotransferase; PT, Prothrombin time; PLT, blood platelet; } \\
\text { CA125, carbohydrate antigen 125; CA199, carbohydrate antigen 199; PTCD, } \\
\text { percutaneous transhepatic cholangial drainage; MRCP, magnetic resonance } \\
\text { cholangiopancreatography. }\end{array}$} \\
\hline
\end{tabular}

Apart from that, univariate Cox's regression analysis revealed that multiple tumors, tumor size lymph node metastases, and previous treatments were related to TTP and OS. Multivariate Cox's regression analysis confirmed the results (Table 3, Table 4).

\section{Subgroup analysis}

Tumor number (Single or Multiple) and lymph node metastasis (yes or no) were selected into subgroup analysis. The results showed that there was no difference of median TTP between DEB-TACE group and cTACE group in patients with single tumor $(P=0.139)$, in patients with multiple tumors $(P=0.370)$, in patients with lymph node metastasis $(P=0.497)$ or in patients with no lymph node metastasis $(P=0.051)$ (Table 5). In patients with multiple tumors or no lymph node metastasis, the median OS of DEB-TACE group was significantly higher than cTACE group $(P=0.032, \quad P=0.023$, respectively). However, DEBTACE group exhibited similar median OS to cTACE group in patients with single tumor $(P=0.107)$ and in patients with lymph node metastasis $(P=0.069)$.

Table 2. Treatment response

\begin{tabular}{|c|c|c|c|}
\hline \multirow[t]{2}{*}{ Response } & \multicolumn{2}{|l|}{ Patients, No. (\%) } & \multirow[t]{2}{*}{$P$ value } \\
\hline & $\overline{\text { DEB-TACE }(\mathrm{N}=40)}$ & cTACE $(\mathrm{N}=49)$ & \\
\hline CR & $0(0.0)$ & $0(0.0)$ & \\
\hline PR & $27(67.5)$ & $28(57.1)$ & \\
\hline SD & $8(20.0)$ & $4(8.2)$ & \\
\hline PD & $5(12.5)$ & $18(36.7)$ & \\
\hline ORR & $27(67.5)$ & $28(57.1)$ & 0.317 \\
\hline DCR & $35(87.5)$ & $32(65.3)$ & 0.011 \\
\hline
\end{tabular}

Table 3. Univariate and multivariate Cox's regression analysis for TTP

\begin{tabular}{|c|c|c|c|c|}
\hline Parameters & $\begin{array}{l}\text { Univariate HR } \\
(95 \% \mathrm{CI})\end{array}$ & $\begin{array}{l}P \\
\text { value }\end{array}$ & $\begin{array}{l}\text { Multivariate } \mathrm{HR}^{\#} \\
(95 \% \mathrm{CI})\end{array}$ & $\begin{array}{l}P \\
\text { value }\end{array}$ \\
\hline $\begin{array}{l}\text { Treatment (cTACE vs. } \\
\text { DEB-TAEC) }\end{array}$ & $1.356(0.888,2.072)$ & 0.158 & $1.386(0.907,2.118)$ & 0.132 \\
\hline Age & $0.987(0.967,1.008)$ & 0.225 & - & - \\
\hline $\begin{array}{l}\text { Gender } \\
\text { (Female vs. male) }\end{array}$ & $1.011(0.659,1.550)$ & 0.960 & - & - \\
\hline HBV (no vs. yes) & $1.445(0.658,3.171)$ & 0.359 & - & - \\
\hline ECOG PS score ( 1 vs. 0$)$ & $1.080(0.701,1.663)$ & 0.728 & - & - \\
\hline $\begin{array}{l}\text { Child-Pugh stage } \\
\text { (B vs. A) }\end{array}$ & $0.819(0.461,1.455)$ & 0.496 & - & - \\
\hline $\begin{array}{l}\text { Tumor number } \\
\text { (multiple vs. single) }\end{array}$ & $1.823(0.733,2.921)$ & 0.034 & $1.819(0.742,2.919)$ & 0.039 \\
\hline Tumor size & $1.706(0.967,1.048)$ & 0.041 & - & - \\
\hline $\begin{array}{l}\text { Lymph node } \\
\text { metastases (yes vs. no) }\end{array}$ & $1.732(0.455,3.179)$ & 0.020 & $1.715(0.445,3.150)$ & 0.047 \\
\hline Ascites (no vs. yes) & $0.829(0.382,1.800)$ & 0.635 & - & - \\
\hline ALT & $0.999(0.995,1.004)$ & 0.741 & - & - \\
\hline Neutrophile & $0.992(0.888,1.109)$ & 0.888 & - & - \\
\hline Lymphocyte & $1.228(0.830,1.816)$ & 0.303 & - & - \\
\hline PLT & $1.001(0.998,1.003)$ & 0.530 & - & - \\
\hline CA125 & $1.000(0.999,1.001)$ & 0.571 & - & - \\
\hline CA199 & $1.000(1.000,1.000)$ & 0.943 & - & - \\
\hline TACE session ( $\geq 2$ vs. 1$)$ & $1.155(0.758,1.761)$ & 0.501 & - & - \\
\hline $\begin{array}{l}\text { Previous treatments } \\
\text { (no vs. yes) }\end{array}$ & $1.782(0.680,2.724)$ & 0.039 & $1.453(0.569,2.349)$ & 0.028 \\
\hline $\begin{array}{l}\text { PTCD/MRCP } \\
\text { (no vs. yes) }\end{array}$ & $1.028(0.652,1.621)$ & 0.906 & - & - \\
\hline \multicolumn{5}{|c|}{$\begin{array}{l}\text { \#variables with } P \text { value } \leq 0.2 \text { in the univariate analysis were further included in the } \\
\text { multivariate Cox proportional hazards regression model analysis. TTP, time to } \\
\text { progression; HR, hazard ratio; CI, confidence interval; cTACE, conventional } \\
\text { transarterial chemoembolization; DEB-TACE, drug-eluting beads transarterial } \\
\text { chemoembolization; HBV, hepatitis B virus; ECOG PS, Eastern Cooperative } \\
\text { Oncology Group Performance Status; ALT, alanine aminotransferase; PLT, blood } \\
\text { platelet; CA125, carbohydrate antigen 125; CA199, carbohydrate antigen 199; } \\
\text { PTCD, percutaneous transhepatic cholangial drainage; MRCP, magnetic resonance } \\
\text { cholangiopancreatography. }\end{array}$} \\
\hline
\end{tabular}

\section{Comparison of adverse events}

In DEB-TACE group, there were $2(5.0 \%), 2$ $(5.0 \%)$ and $1(2.5 \%)$ patient experienced Inguinal 
hematoma, hepatic arterial dissection and hepatorenal syndrome, respectively (Table 6). While in cTACE group, $2(4.1 \%), 1(2.0 \%)$ and $0(0.0 \%)$ patients experienced Inguinal hematoma, hepatic arterial dissection and hepatorenal syndrome. There was no difference of adverse event rate between DEB-TACE group and cTACE group (all $P>0.05$ ). Liver function (bilirubin, albumin, and PT) were evaluated at 4 weeks after the first TACE (DEB-TACE, cTACE) treatment, and did not differ significantly from the baseline values at this time point (Figure 3).

Table 4. Univariate and multivariate Cox's regression analysis for OS

\begin{tabular}{|c|c|c|c|c|}
\hline Parameters & $\begin{array}{l}\text { Univariate HR } \\
(95 \% \mathrm{CI})\end{array}$ & $\begin{array}{l}P \\
\text { value }\end{array}$ & $\begin{array}{l}\text { Multivariate } \mathrm{HR}^{\#} \\
(95 \% \mathrm{CI})\end{array}$ & $\begin{array}{l}P \\
\text { value }\end{array}$ \\
\hline $\begin{array}{l}\text { Treatment (cTACE vs. } \\
\text { DEB-TAEC) }\end{array}$ & $1.763(1.131,2.749)$ & 0.012 & $1.654(1.048,2.608)$ & 0.031 \\
\hline Age & $0.986(0.966,1.007)$ & 0.203 & - & - \\
\hline $\begin{array}{l}\text { Gender (Female vs. } \\
\text { male) }\end{array}$ & $1.031(0.665,1.599)$ & 0.891 & - & - \\
\hline HBV (no vs. yes) & $1.185(0.540,2.604)$ & 0.672 & - & - \\
\hline ECOG PS score ( 1 vs. 0 ) & $1.343(0.859,2.099)$ & 0.196 & $1.326(0.846,2.078)$ & 0.219 \\
\hline $\begin{array}{l}\text { Child-Pugh stage (B vs. } \\
\text { A) }\end{array}$ & $0.929(0.521,1.658)$ & 0.804 & - & - \\
\hline $\begin{array}{l}\text { Tumor number } \\
\text { (multiple vs. single) }\end{array}$ & $1.997(0.711,2.993)$ & 0.025 & $1.852(0.690,2.895)$ & 0.037 \\
\hline Tumor size & $1.820(0.979,2.563)$ & 0.042 & $1.734(0.832,2.498)$ & 0.045 \\
\hline $\begin{array}{l}\text { Lymph node } \\
\text { metastases (no vs. yes) }\end{array}$ & $0.829(0.524,1.312)$ & 0.424 & - & - \\
\hline Ascites (no vs. yes) & $0.704(0.322,1.539)$ & 0.379 & - & - \\
\hline ALT & $1.000(0.996,1.005)$ & 0.825 & - & - \\
\hline Neutrophile & $1.015(0.907,1.137)$ & 0.792 & - & - \\
\hline Lymphocyte & $1.311(0.887,1.937)$ & 0.175 & $1.213(0.808,1.820)$ & 0.352 \\
\hline PLT & $1.001(0.998,1.003)$ & 0.697 & - & - \\
\hline CA125 & $1.000(0.999,1.001)$ & 0.645 & - & - \\
\hline CA199 & $1.000(1.000,1.000)$ & 0.991 & - & - \\
\hline TACE session ( $\geq 2$ vs. 1 ) & $1.150(0.748,1.766)$ & 0.524 & - & - \\
\hline $\begin{array}{l}\text { Previous treatments } \\
\text { (no vs. yes) }\end{array}$ & $1.809(0.751,2.946)$ & 0.034 & $1.794(0.742,2.894)$ & 0.042 \\
\hline $\begin{array}{l}\text { PTCD/MRCP } \\
\text { (no vs. yes) }\end{array}$ & $1.218(0.756,1.961)$ & 0.417 & - & - \\
\hline
\end{tabular}

\#variables with $P$ value $\leq 0.2$ in the univariate analysis were further included in the multivariate Cox proportional hazards regression model analysis. OS, overall survival; $\mathrm{HR}$, hazard ratio; CI, confidence interval; cTACE, conventional transarterial chemoembolization; DEB-TACE, drug-eluting beads transarterial chemoembolization; HBV, hepatitis B virus; ECOG PS, Eastern Cooperative Oncology Group Performance Status; ALT, alanine aminotransferase; PLT, blood platelet; CA125, carbohydrate antigen 125; CA199, carbohydrate antigen 199; PTCD, percutaneous transhepatic cholangial drainage; $\mathrm{MRCP}$, magnetic resonance cholangiopancreatography.

Table 5. Subgroup analysis of TTP and OS based on tumor features

\begin{tabular}{lllc}
\hline Items & DEB-TACE & cTACE & $P$ value \\
\hline Single tumor & $\mathrm{n}=16$ & $\mathrm{n}=23$ & \\
Median TTP $(95 \% \mathrm{CI})$, months & $6(4.7,7.3)$ & $2(0.7,3.3)$ & 0.139 \\
Median OS $(95 \% \mathrm{CI})$, months & $14(12.7,15.3)$ & $6(4.0,8.0)$ & 0.107 \\
Multiple tumors & $\mathrm{n}=24$ & $\mathrm{n}=26$ & \\
Median TTP $(95 \% \mathrm{CI})$, months & $4(3.1,4.9)$ & $2(1.0,3.0)$ & 0.370 \\
Median OS $(95 \% \mathrm{CI})$, months & $9(7.1,10.9)$ & $6(4.3,7.7)$ & 0.032 \\
Lymph node metastasis & $\mathrm{n}=31$ & $\mathrm{n}=29$ & \\
Median TTP $(95 \% \mathrm{CI})$, months & $4(2.0,6.0)$ & $3(2.1,3.9)$ & 0.497 \\
Median OS $(95 \% \mathrm{CI})$, months & $9(4.6,13.4)$ & $6(4.2,7.8)$ & 0.069 \\
No lymph node metastasis & $\mathrm{n}=9$ & $\mathrm{n}=20$ & \\
Median TTP $(95 \% \mathrm{CI})$, months & $6(2.1,8.9)$ & $2(0.6,3.4)$ & 0.051 \\
Median OS (95\%CI), months & $17(12.8,21.2)$ & $6(3.8,8.2)$ & 0.023 \\
\hline $\begin{array}{l}\text { TTP, time to progression; OS, overall survival; DEB-TACE, drug-eluting beads } \\
\text { transarterial chemoembolization; cTACE, conventional transarterial }\end{array}$ \\
chemoembolization.
\end{tabular}

Table 6. Adverse events after TACE treatment

\begin{tabular}{llll}
\hline Adverse events & $\begin{array}{l}\text { DEB-TACE (N } \\
=40)\end{array}$ & $\begin{array}{l}\text { c-TACE } \\
(\mathrm{N}=49)\end{array}$ & $\begin{array}{l}P \\
\text { value }\end{array}$ \\
\hline Vomiting/Nause & $28(70.0)$ & $35(71.4)$ & 0.883 \\
Abdominal pain & $25(62.5)$ & $42(85.7)$ & 0.012 \\
fever & $16(40.0)$ & $26(53.1)$ & 0.220 \\
Inguinal hematoma, No. (\%) & $2(5.0)$ & $2(4.1)$ & 0.836 \\
Hepatic arterial dissection, No. (\%) & $2(5.0)$ & $1(2.0)$ & 0.441 \\
Hepatorenal syndrome, No. (\%) & $1(2.5)$ & $0(0.0)$ & 0.204 \\
Pulmonary oil embolization, No. (\%) & $0(0.0)$ & $0(0.0)$ & - \\
\hline $\begin{array}{l}\text { TACE,transarterial chemoembolization; DEB-TACE, drug-eluting beads } \\
\text { transarterial chemoembolization; cTACE, conventional transarterial }\end{array}$ \\
chemoembolization.
\end{tabular}

A

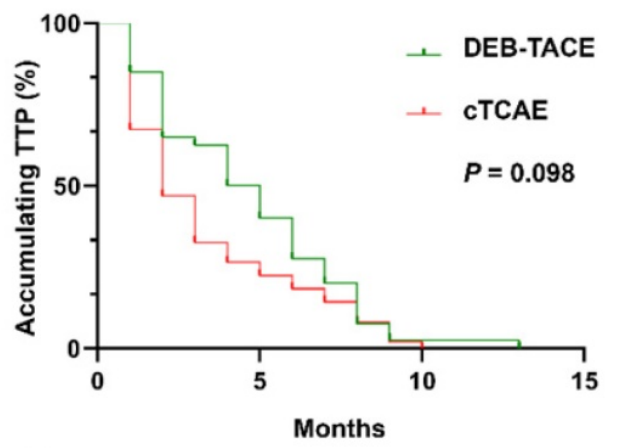

Number at risk

$\begin{array}{lllll}\text { DEB-TACE } & 40 & 20 & 3 & 0 \\ \text { cTCAE } & 49 & 13 & 1 & 0\end{array}$

B
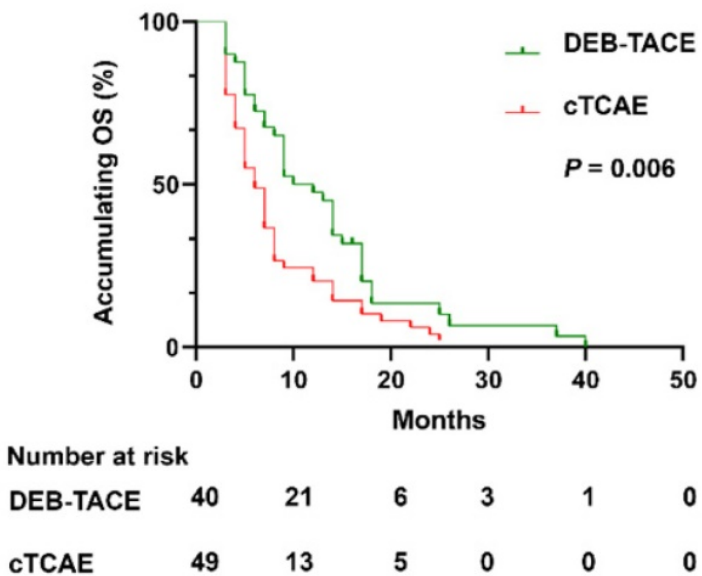

Figure 2. DEB-TACE achieved longer OS but similar TTP compared to cTACE. DEB-TACE achieved similar accumulating TTP (A), while prolonged OS (B) compared to cTACE. DEB-TACE: Drug-eluting beads transarterial chemoembolization, OS: overall survival, TTP: time to progression, cTACE: conventional transarterial chemoembolization, ICC: intrahepatic cholangiocarcinoma. 

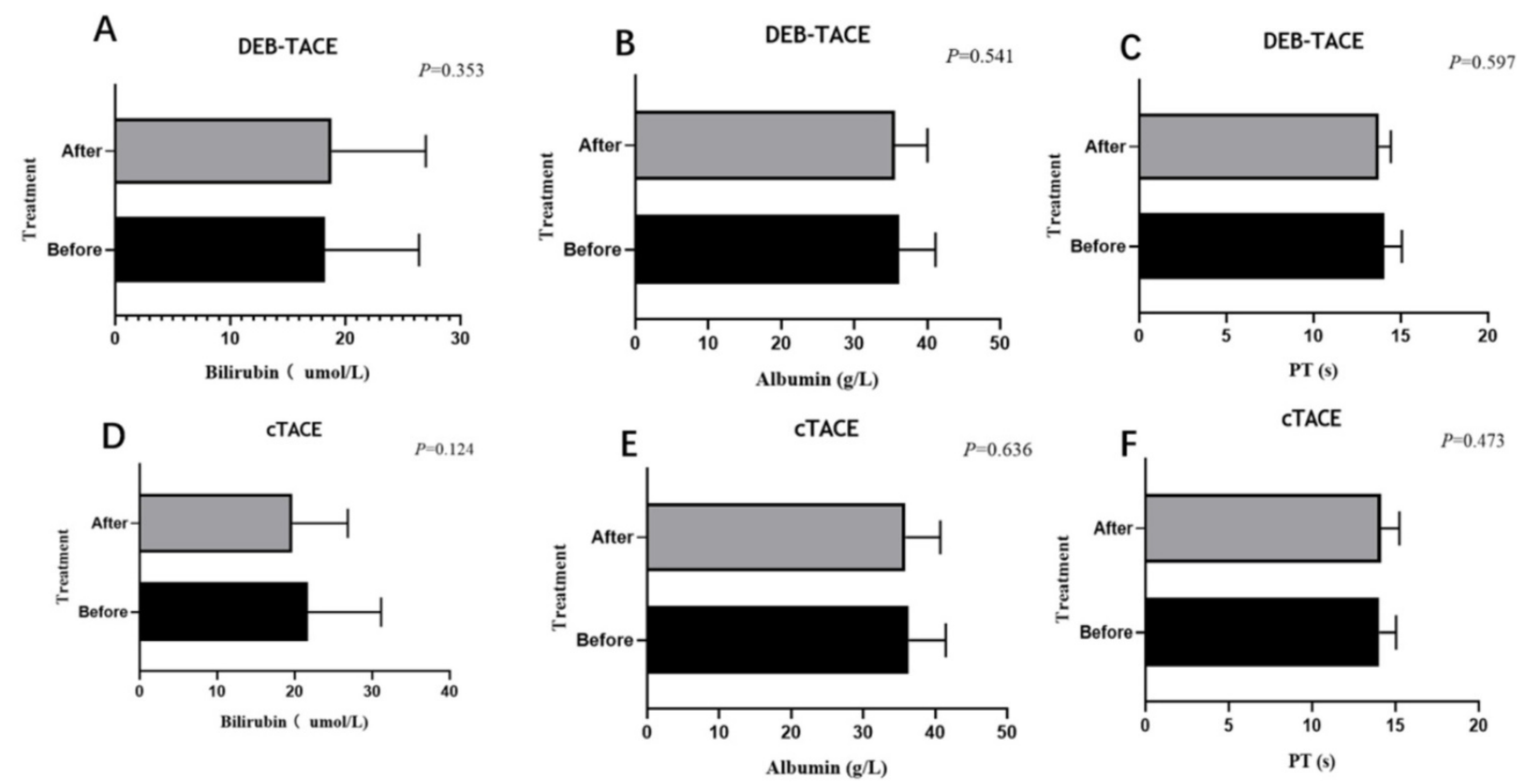

Figure 3. Liver function before and after (4 weeks) the treatment. Liver function including albumin (A, D), bilirubin (B, E), PT (C, F) of the DEB-TACE group or cTACE group before and after treatment at 4 weeks. Paired sample T-test showed no statistic difference $(P>0.05)$.

\section{Discussion}

DEB-TACE is widely used technique and gains huge attention in treating patients with liver malignancy [6]. It is reported that DEB-TACE with Callispheres microsphere increases tumor response featured by higher ORR and CR rates compared to CTACE in HCC patients $[9,10]$. Regarding its clinical application in ICC patients, two single-armed retrospective studies show that the ORR rates in DEB-TACE with Callispheres microsphere treated ICC patients are $65.9 \%$ and $67.6 \%$, respectively $[12,13]$. Since DEB-TACE with Callispheres microsphere displays improved efficacy and safe compared to cTACE in treating HCC patients, also DEB-TACE with Callispheres microsphere is safe and well-tolerant in ICC patients, therefore we hypothesized that DEB-TACE with Callispheres microsphere might achieve great efficacy compared to cTACE in ICC patients. However, no relevant study has been conducted. Thus, we performed this study and discovered that DEB-TACE with Callispheres microsphere therapy resulted in higher DCR compared to CTACE in treating ICC patients which could be explained as follows: DEB-TACE increased intratumoural concentration and released loaded chemotherapeutic agent in a controlled manner, therefore resulted in increased target tumor necrosis and further led to enhanced tumor response (featured by DCR in the present study) in ICC patients $[6,7,8]$.

Apart from the comparison of DEB-TACE with Callispheres microsphere versus CTACE in term of their short-term efficacy, the comparison of these two TACE techniques regarding the long-term survival profile is also of great interest. However, no relevant study has been performed. In the present study, we discovered that DEB-TACE with Callispheres microsphere resulted in prolonged OS in ICC patients compared to CTACE, also it could independently predict longer OS. Furthermore, DEB-TACE with Callispheres microsphere achieved longer OS compared to CTACE in ICC patients with multiple tumors and patients with no lymph node metastasis. The possible reasons to explain these results were: (a) DEB-TACE increased tumor necrosis and enhanced tumor response as mentioned earlier, therefore led to reduced recurrence risk and favorable survival profile in ICC patients $[6,7,8]$. (b) Regarding multiple tumors, it might indicate various cellular origins and molecular evolutions, which made them difficult to treat [15]. While by using DEB-TACE, multiple tumors were easier to embolism compared with CTACE, which led to favorable prognosis in ICC patients [6]. (c) Regarding lymph node metastasis, it suggested distal tumor formation and growth through the lymphatic vessels, therefore led to more advanced stage of ICC and increased mortality risk, which eventually resulted in unfavorable survival profile in ICC patients [14]. Taken together, ICC patients underwent DEB-TACE with Callispheres microsphere achieved favorable survival profile than cTACE.

In terms of the safety profile of DEB-TACE with Callispheres microsphere in ICC patients, one study 
discovers that the major adverse events of DEB-TACE with Callispheres microsphere in ICC patients are abdominal pain, fever, vomiting and nausea, which are all mild and manageable [12]. Among these, patients received DEB-TACE significantly less likely to experience abdominal pain compared to patients received CTACE. Another single-armed retrospective study shows that DEB-TACE with Callispheres microsphere induces similar adverse events (including pain, fever, vomiting and nausea) in ICC patients, suggesting that DEB-TACE with Callispheres microsphere is relative safe in treating ICC patients [13]. In this double-armed comparative study, we discovered that the main adverse events in ICC patients related to DEB-TACE with Callispheres microsphere were inguinal hematoma, hepatic arterial dissection and hepatorenal syndrome, suggesting the relative safe profile of DEB-TACE with Callispheres microsphere. Although the load of chemotherapy drugs in DEB-TACE is higher, there was no difference of adverse event rates between DEB-TACE with Callispheres microsphere and CTACE in ICC patients, which could be explained that DEB-TACE reduced systematic toxicity by preventing loaded antitumor agent leakage compared to cTACE $[6,7,8]$.

Several limitations existed in the current study. Firstly, the sample size in our study was relatively small, therefore a further study with larger sample size to validate the safety and efficacy of DEB-TACE with Callispheres microsphere in unresectable ICC patients was necessary. Secondly, as a real-world study, some compounding factors might occur, therefore further randomized controlled trial (RCT) validation was needed. Finally, this was a single-center study, which might cause selection bias of patients, and further study with multiple centers was necessary.

\section{Conclusions}

In conclusion, DEB-TACE with Callispheres microsphere is superior to CTACE in treating unresectable ICC patients regarding its better efficacy and equal safety.

\section{Abbreviations}

ICC: intrahepatic Cholangiocarcinoma; DEBTACE: drug-eluting beads transarterial chemoembolization; c-TACE: conventional TACE; TTP: the median time to progression; OS: overall survival; DCR: disease control rate; mRECIST: the modified Response Evaluation Criteria in Solid Tumors; HCC: hepatocellular carcinoma; ECOG: Eastern Cooperative Group Performance Status; CT: computed tomography; MRI: magnetic resonance image; CR: complete response; PR: partial response;
SD: stable disease; PD: progressive disease.

\section{Acknowledgements}

This study was supported by the National Natural Science Foundation of China (No. 81873919 and No. 81801810).

\section{Ethics statement}

This study protocols were approved by the Ethics Committee of Wuhan union hospital

\section{Competing Interests}

The authors have declared that no competing interest exists.

\section{References}

1. Zhang $\mathrm{H}$, Yang $\mathrm{T}, \mathrm{Wu} \mathrm{M}$, et al. Intrahepatic cholangiocarcinoma: Epidemiology, risk factors, diagnosis and surgical management. Cancer Lett. 2016; 379:198-205.

2. El-Diwany R, Pawlik TM, Ejaz A, et al. Intrahepatic Cholangiocarcinoma. Surg Oncol Clin N Am. 2019; 28:587-599.

3. Rizvi S, Khan SA, Hallemeier CL, et al. Cholangiocarcinoma - evolving concepts and therapeutic strategies. Nat Rev Clin Oncol. 2018; 15:95-111.

4. Lee AJ, Chun YS, et al. Intrahepatic cholangiocarcinoma: the AJCC/UICC 8th edition updates. Chin Clin Oncol. 2018; 7:52.

5. Savic LJ, Chapiro J, Geschwind JH, et al. Intra-arterial embolotherapy for intrahepatic cholangiocarcinoma: update and future prospects. Hepatobiliary Surg Nutr. 2017; 6:7-21

6. Tsurusaki M, Murakami T, et al. Surgical and Locoregional Therapy of HCC: TACE. Liver Cancer. 2015; 4:165-75.

7. Chang Y, Jeong SW, Young Jang J, et al. Recent Updates of Transarterial Chemoembolilzation in Hepatocellular Carcinoma. Int J Mol Sci. 2020; 21:8165.

8. Facciorusso A, et al. Drug-eluting beads transarterial chemoembolization for hepatocellular carcinoma: Current state of the art. World J Gastroenterol. 2018; 24:161-169.

9. Ma Y, Zhao C, Zhao H, Li H, et al. Comparison of treatment efficacy and safety between drug-eluting bead transarterial chemoembolization with CalliSpheres ${ }^{\circledR}$ microspheres and conventional transarterial chemoembolization as first-line treatment in hepatocellular carcinoma patients. Am J Transl Res. 2019; 11:7456-7470.

10. Zhao C, Ma SPZCY, et al. Comparison of treatment response, survival and safety between drug-eluting bead transarterial chemoembolization with CalliSpheres ${ }^{\circledR}$ microspheres versus conventional transarterial chemoembolization in treating hepatocellular carcinoma. J BUON. 2019; 24:1150-1166.

11. Li H, Wu F, Duan M, Zhang G, et al. Drug-eluting bead transarterial chemoembolization (TACE) vs conventional TACE in treating hepatocellular carcinoma patients with multiple conventional TACE treatments history: A comparison of efficacy and safety. Medicine (Baltimore). 2019; 98:e15314.

12. Luo J, Zheng J, Shi $\mathrm{C}$, et al. Drug-eluting beads transarterial chemoembolization by CalliSpheres is effective and well tolerated in treating intrahepatic cholangiocarcinoma patients: A preliminary result from CTILC study. Medicine (Baltimore). 2020; 99:e19276.

13. Zhou TY, Zhou GH, Zhang YL, et al. Drug-eluting beads transarterial chemoembolization with Callispheres microspheres for treatment of unresectable intrahepatic cholangiocarcinoma. J Cancer. 2020; 11:4534-4541.

14. Suhail Y, Cain MP, Vanaja K, et al. Systems Biology of Cancer Metastasis. Cell Syst. 2019; 9:109-127.

15. Wei M, Lü L, Lin P, et al. Multiple cellular origins and molecular evolution of intrahepatic cholangiocarcinoma. Cancer Lett. 2016; 379:253-61.

16. Bridgewater J, Galle PR, Khan SA, et al. Guidelines for the diagnosis and management of intrahepatic cholangiocarcinoma. J Hepatol. 2014; 60:1268-89.

17. Han $X$, Chen $Q$, Sun $Y$, et al. Morphology, Loadability, and Releasing Profiles of CalliSpheres Microspheres in Delivering Oxaliplatin: An In vitro Study. Technol Cancer Res Treat. 2019; 18:1533033819877989. 